\title{
Scanning Tunneling Microscopy and Spectroscopy on Iron-Pnictides
}

\section{Citation}

Yin, Yi, Martin Zech, Tess L. Williams, and Jennifer E. Hoffman. 2009. Physica C:

Superconductivity 469(9-12): 535-544.

\section{Published Version}

http://dx.doi.org/10.1016/j.physc.2009.03.053

\section{Permanent link}

http://nrs.harvard.edu/urn-3:HUL.InstRepos:3009615

\section{Terms of Use}

This article was downloaded from Harvard University's DASH repository, and is made available under the terms and conditions applicable to Open Access Policy Articles, as set forth at http:// nrs.harvard.edu/urn-3:HUL.InstRepos:dash.current.terms-of-use\#OAP

\section{Share Your Story}

The Harvard community has made this article openly available.

Please share how this access benefits you. Submit a story.

Accessibility 


\title{
Scanning Tunneling Microscopy and Spectroscopy on Iron-Pnictides
}

\author{
Yi Yin, M. Zech, T. L. Williams, J. E. Hoffman \\ Harvard University, Department of Physics, Cambridge, MA 02138
}

\begin{abstract}
Tremendous excitement has followed the recent discovery of superconductivity up to $T_{c}=$ $56 \mathrm{~K}$ in iron-arsenic based materials (pnictides). This discovery breaks the monopoly on high- $T_{c}$ superconductivity held by copper-oxides (cuprates) for over two decades and renews hope that high- $T_{c}$ superconductivity may finally be theoretically understood and widely applied.

Since scanning tunneling microscopy (STM) and spectroscopy (STS) have been key tools in the investigation and understanding of both conventional and unconventional superconductivity, these techniques are also applied to the pnictides. While the field is still in its early stages, several important achievements by STM and STS have been reported on the pnictides. In this paper, we will review their contribution towards an understanding of superconductivity in this new class of materials.
\end{abstract}

Key words: Pnictides, Superconductivity, Scanning Tunneling Microscopy, Vortex Imaging PACS: 68.37.Ef, 74.25.Qt, 74.25.Jb, 74.50.+r

\section{Introduction}

One of the most exciting events in condensed matter physics in 2008 was the discovery of superconductivity in the ironarsenic based compounds (pnictides) $[1,2$, $3,4,5,6]$. After the initial fluorine doped $\mathrm{LaFeAsO}_{1-x} \mathrm{~F}_{x}$ with a superconducting transition temperature of $T_{\mathrm{c}}=26 \mathrm{~K}[1]$, superconductors with $T_{\mathrm{c}}$ up to $56 \mathrm{~K}$ have been obtained by replacing lanthanum with other rare earth elements of smaller ionic radii $[3,4,5]$ or by Th doping [7]. Due to their stoichiometry, these compounds are referred to as 1111 type. Subsequently,

\footnotetext{
Email address:

jhoffman@physics.harvard.edu (J. E. Hoffman) Preprint submitted to Physica $C$
}

so-called 122 type, non-oxides $\mathrm{AFe}_{2} \mathrm{As}_{2}$ $(\mathrm{A}=\mathrm{Ba}, \mathrm{Sr}, \mathrm{Ca}, \mathrm{Eu})$ were also found to be superconducting when appropriately doped or pressurized $[8,9,10,11,12,13,14,15]$. Both 1111 and 122 unit cells contain an FeAs layer, implying that superconductivity emerges out of this layer in the pnictides.

Like the cuprates, the pnictides have a layered structure. Consequently, highquality single crystals can be mechanically cleaved to obtain atomically flat and clean surfaces suitable for characterization by surface sensitive probes such as scanning tunneling microscopy (STM) and spectroscopy (STS). This cleaving procedure, however, cannot be applied to samples less than $\sim 100 \mu \mathrm{m}$ in size. To date, the largest sinMay 23, 2009 
gle crystals, reaching $3 \times 5 \times 0.2 \mathrm{~mm}^{3}$ in size, are members of the non-oxide 122 family, grown with Sn or FeAs flux $[9,16]$. In contrast, only tiny single crystals of the 1111 family exist, as large as $200 \times 100 \times 30 \mu \mathrm{m}^{3}$ $[17,18]$. Therefore, most of the STM/STS studies have been carried out on $\mathrm{Ba} / \mathrm{Sr} 122$ samples, with only a few STS measurements on 1111 poly-crystals.

The 122 family of pnictides becomes superconducting upon the introduction of either hole or electron dopants. For example, $\mathrm{BaFe}_{2} \mathrm{As}_{2}$ becomes a hole-doped superconductor upon replacement of $\mathrm{Ba}^{2+}$ by $\mathrm{K}^{+}[8]$, or an electron-doped superconductor upon replacement of $\mathrm{Fe}^{2+}$ by $\mathrm{Co}^{3+}$ or $\mathrm{Ni}^{4+}[19,20]$. The emergence of superconductivity through chemical substitution directly into the superconducting layer is in stark contrast with the cuprates, where the substitution of impurity elements for even a small percentage of the $\mathrm{Cu}$ atoms can destroy superconductivity [21].

Low temperature STM and STS have already proven to be important tools for the investigation of cuprates, since they are capable of probing the electronic density of states (DOS) with atomic resolution [22]. This information is crucial because the local DOS provides direct information about the superconducting electronic structure, its spatial inhomogeneity $[23,24,25,26]$, its behavior in proximity to impurities [27, 28, 29], its doping dependence [25] and its magnetic field dependence $[30,31,32,33,34]$. This success motivates the application of STM and STS to the pnictides to gain insight to the underlying mechanism of superconductivity in these compounds.

In this review article, we will summarize STM and STS investigations of pnictide superconductors during the first year after their discovery. After an introduction to low temperature STM in Section 2, we will discuss the crystal structure and surface characterization of the pnictides in Section 3. Then we will focus on STS measurements of the pnictides: superconducting spectra and spectral maps in Section 4 and vortex imaging in Section 5. In Section 6, we conclude with suggestions for future STM/STS experiments that could shed additional light on these materials.

\section{STM Techniques}

An STM consists of a sharp metallic tip which is scanned across an electrically conducting sample surface at very small separation, typically several angstroms. Both tipsample separation and lateral position can be varied with sub- $\AA$ precision by means of a piezoelectric scanner (see Fig. 1). When a negative bias voltage is applied to the sample, electrons in occupied states of the sample will tunnel through the vacuum barrier to the empty states of the tip, resulting in a measurable tunneling current. A positive bias voltage applied to the sample will likewise cause electrons to tunnel from tip to sample. The tunneling current $I$ depends exponentially on the tip-sample distance $d[35]$ according to

$$
I \propto e^{-2 \kappa d} ; \quad \kappa=\sqrt{2 m \phi} / \hbar
$$

where $\phi$ is the average work function of tip and sample; the work function measured by STM is usually of order $3 \mathrm{eV}$ in metals. Materials commonly used as STM tips, chosen for featureless DOS near the Fermi energy $E_{\mathrm{F}}$, are platinum $(\mathrm{Pt})$, platinumiridium (PtIr) alloys, and tungsten (W).

If the tunneling current is kept constant while the STM tip is scanned over the sample surface, a contour of constant integrated 
DOS is followed. This technique is referred to as constant-current mode, and in the case of homogeneous elemental metals the contour of constant DOS corresponds to the topography of the sample surface. If the local DOS varies spatially, the resulting image contains a mixture of DOS and topographic information. With the appropriate tunneling parameters, however, the contribution of the topography dominates the image.

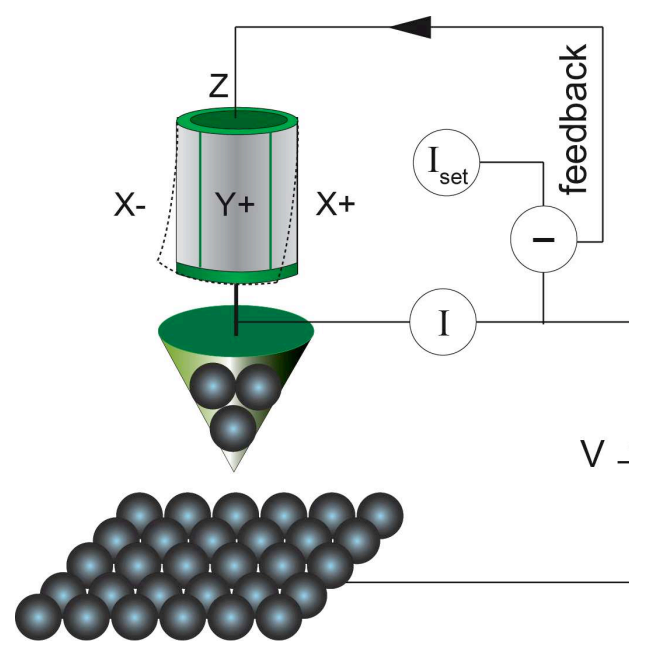

Figure 1: Schematic of an STM. A voltage is applied between the conducting sample surface and a sharp metallic tip, leading to a measurable tunneling current with exponential dependence on the tip-sample separation. If this current is kept constant while the STM tip is scanned over the surface, the surface topography and/or variation in the local DOS can be revealed.

In addition to revealing the geometrical surface structure of a sample, STM can also measure the sample DOS as a function of energy, up to several eV from the Fermi level in occupied and unoccupied states. This is usually done by sweeping the bias voltage $V$ and measuring the tunneling current $I$ while maintaining constant tip-sample separation $d$. By numerically differentiating $I(V)$, the conductance $d I / d V$ can be obtained. To re- duce noise, a lock-in technique can be used: a small AC modulation is summed with the bias voltage and the tunneling current is demodulated to yield $d I / d V$. The interpretation of $d I / d V$ spectra can be complex but it can be shown that, under ideal conditions, $d I / d V$ is a good measure of the sample DOS.

If these $d I / d V$ spectra are recorded on a dense array of locations in real space, spatial variation in the sample DOS can be extracted. This is referred to as DOSmapping. Applications of this technique include the measurement of local gap variations and imaging of vortices in magnetic field. The magnitude $\Delta$ of the gap can be estimated by halving the distance between the coherence peaks. A "gap map" can be obtained by plotting $\Delta$ for every spectrum within a DOS map.

STM has been applied with great success to cuprate superconductors, addressing pairing symmetry, gap inhomogeneity, dopant placement, and vortex pinning [22]. STM is a tool unique in its ability to provide atomic-resolution real-space maps of the electronic density of states. From a practical standpoint, STM is also ideally suited to study new layered superconducting materials which may be initially available only in small single crystals.

\section{Crystal Structure and Surface Characterization}

Pnictides are layered compounds which can be mechanically cleaved to expose the $a b$-plane (see Fig. 2). Consequently, all of the STM/STS studies on cleaved single crystal pnictides so far are taken along the (001) direction, but the exact composition of the exposed surface is still an open question. In the cuprate superconductor most 
widely studied by $\mathrm{STM}, \mathrm{Bi}_{2} \mathrm{Sr}_{2} \mathrm{CaCu}_{2} \mathrm{O}_{8+\delta}$ (Bi2212), cleaving usually occurs between mirror $\mathrm{BiO}$ planes coupled by weak van der Waals forces [22], removing a complete $\mathrm{BiO}$ layer and leaving behind a chargeneutral surface. Among known pnictide superconductors, no such natural, chargebalanced cleavage planes exist. Pnictide cleaving recalls the cuprate superconductor $\mathrm{YBa}_{2} \mathrm{Cu}_{3} \mathrm{O}_{7-\delta}(\mathrm{Y} 123)$, which cleaves between $\mathrm{BaO}$ and $\mathrm{CuO}$ chain layers without neutral exposed planes [36].

For 1111 pnictides such as LaFeAsO, the inter-coupling between $(\mathrm{LaO})^{+}$and $(\mathrm{FeAs})^{-}$ layers is mostly ionic, but weak compared to the ionic bonding within $\mathrm{LaO}$ layers and the covalent bonding within FeAs layers $[37,38]$. Thus we expect LaFeAsO crystals to cleave between $\mathrm{LaO}$ and FeAs layers, exposing a charged surface plane. This expectation is substantiated by angle resolved photoemission spectroscopy (ARPES) measurements of the enclosed Fermi surface volume on the cleaved surface of isostructural LaFePO [39].

When studying materials that have a polar cleaved surface, the electronic structure detected using surface-sensitive techniques is likely to be different from that of the bulk, affecting measurements of carrier density, Fermi level, and effective doping [40]. In addition, atomic reconstruction, adsorption of charged contaminants, and electronic reconstruction might occur at the surface in order to compensate for the charge imbalance [41].

In 122 pnictides, $\mathrm{A}^{2+}(\mathrm{A}=\mathrm{Ba}, \mathrm{Sr}, \mathrm{Ca}, \mathrm{Eu})$ sheets are sandwiched between (FeAs) ${ }^{-}$layers. In this case, both inter-plane bonding and $\mathrm{A}^{2+}$ in-plane bonding are relatively weak compared to the covalent FeAs bonding. Therefore, crystals are likely to cleave either between $\mathrm{A}^{2+}$ and $(\mathrm{FeAs})^{-}$planes,
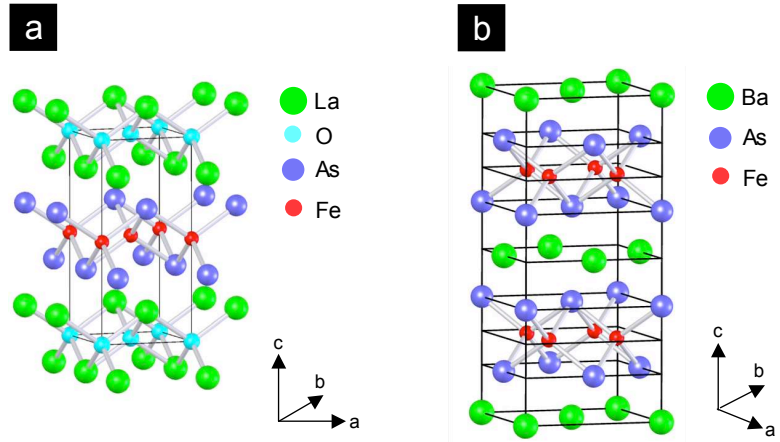

Figure 2: Crystal structure of the pnictides. (a) Tetragonal LaFeAsO 1111 type with room temperature lattice constants $\mathrm{a}=\mathrm{b}=4.03 \AA$ and $\mathrm{c}=$ $8.73 \AA$ [1]. (b) Tetragonal $\mathrm{BaFe}_{2} \mathrm{As}_{2} 122$ type with room temperature lattice constants $\mathrm{a}=\mathrm{b}=3.96 \AA$ and $\mathrm{c}=12.39 \AA$ [42]. The FeAs layer is the effective superconducting layer in both compounds.

leaving behind a charged surface, or within the $\mathrm{A}^{2+}$ plane, leaving about half of the $\mathrm{A}^{2+}$ atoms on each of the exposed, charge neutral surfaces. The predominance of the latter scenario is substantiated by Fermi surface volume measurements from ARPES studies of $\mathrm{BaFe}_{2} \mathrm{As}_{2}[43,44]$.

On $\mathrm{Sr}_{1-x} \mathrm{~K}_{x}$ FeAs with $T_{\mathrm{c}}=32 \mathrm{~K}$, cleaved at $10 \mathrm{~K}$, Boyer et al. found a strong $2 \times 1$ stripe feature modulating a square atomic lattice with spacing $a_{0}=4.0 \AA$ along the stripe direction (Fig. 3a) [45]. This atomic spacing is compatible with the lattice constant $a_{0}$ of either $\mathrm{Sr}$ or As planes [42]. Serendipitously, in a small number of areas the next atomic plane is exposed, displaying a square lattice shifted by half a unit cell with respect to the atoms along the stripes. This finding leads Boyer et al. to conclude that the stripes are a surface reconstruction of a nearly complete $\mathrm{Sr} / \mathrm{K}$ layer sitting on top of an FeAs layer.

A $2 \times 1$ stripe feature has also been observed by Yin et al. [46] and by Massee et 

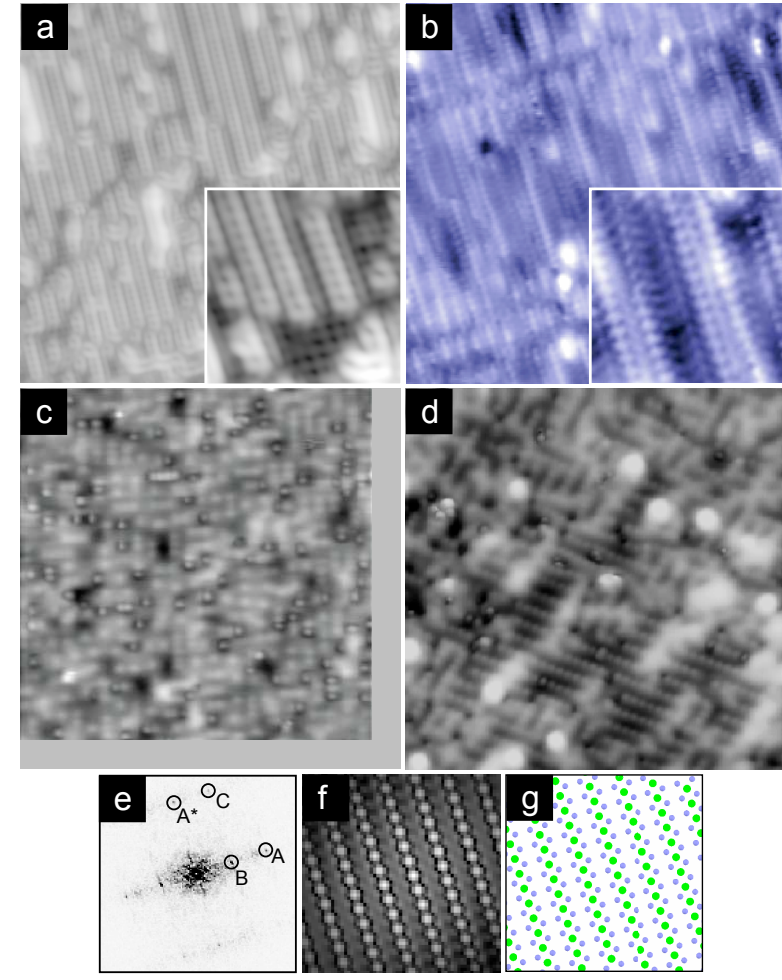

Figure 3: Topographic images of different single crystalline 122 compounds as recorded by four groups, revealing stripe-like surface features. (a) $20 \times 20 \mathrm{~nm}^{2}$ constant-current topography of $\mathrm{Sr}_{1-x} \mathrm{~K}_{x} \mathrm{Fe}_{2} \mathrm{As}_{2}$ by Boyer et al., cold-cleaved and recorded at $5.3 \mathrm{~K}[45]$. The inset shows a $5 \times 5 \mathrm{~nm}^{2}$ zoom. (b) $20 \times 20 \mathrm{~nm}^{2}$ constant-current topography of $\mathrm{BaFe}_{1.8} \mathrm{Co}_{0.2} \mathrm{As}_{2}$ by Yin et al., cold-cleaved and recorded at $6.25 \mathrm{~K}$ [46]. The inset shows a $5 \times 5 \mathrm{~nm}^{2}$ zoom. (c) $18.9 \times 18.9 \mathrm{~nm}^{2}$ constantcurrent topography of $\mathrm{BaFe}_{1.86} \mathrm{Co}_{0.14} \mathrm{As}_{2}$ by Massee et al., cleaved at room temperature and recorded at $4.2 \mathrm{~K}$ [47]. (d) $20 \times 20 \mathrm{~nm}^{2}$ constant-current topography of $\mathrm{SrFe}_{2} \mathrm{As}_{2}$ by Hsieh et al., cleaved at room temperature and recorded at $40 \mathrm{~K}[48]$. (e) Fourier transformation of the topographic image in (b). The main features are a central high-intensity area representing long length scale inhomogeneity, and 4 distinct points (with their symmetric equivalents): $A$ and $A^{\star}$ result from atomic spacing between and along the stripes, respectively; $B$ results from the alternating stripe intensity; $C$ results from the shift of atoms along alternate rows. (f) Reverse Fourier transformation of (e) after filtering out the central high-intensity area, demonstrating more clearly the atomic locations of the original topography without the longer wavelength disorder.
Image clearly suggests that alternate rows of $\mathrm{Ba}$ have been removed, exposing the shifted rows of As atoms beneath. (g) Schematic of the surface after cleaving scenario suggested by (f). Large green dots correspond to Ba atoms, small purple ones to As atoms. (f) and (g) are the same scale as the inset in (b).

and $22 \mathrm{~K}$, respectively. Yin et al., after cleaving at $T \leq 30 \mathrm{~K}$, have observed a complex unit-cell-doubling stripe feature, where alternate rows display contrasting brightness and clarity, as well as atomic shifts by approximately half a lattice constant along the row direction, demonstrated in Fig. 3b. The alternating row intensity may be attributed to either height or density of states variations. This complex surface structure could be explained by the removal of every second row of $\mathrm{Ba}$ atoms by cleaving. This scenario is supported by Fourier transform analysis of the topography (see Fig. 3e-f): the less distinct, shifted rows (see inset of Fig. $3 \mathrm{~b}$ ) would consist of two rows of underlying As atoms between each row of $\mathrm{Ba}$ atoms. This is also supported by increasing evidence from ARPES measurements, which indicate that 122 compounds predominantly cleave charge neutral $[43,44]$.

Massee et al., cleaving $\mathrm{BaFe}_{1.86} \mathrm{Co}_{0.14} \mathrm{As}_{2}$ at room temperature, also report stripe features with an average separation of $\sim 8 \AA$, shown in Fig. 3c. Within some rows, single atoms are resolved, revealing an in-row spacing of $3.9 \AA$. Massee et al. emphasize that details of the topography vary from cleave to cleave and that sometimes only $2 \mathrm{D}$ maze-like networks are present.

The stripe feature is also reported in the parent compound $\mathrm{SrFe}_{2} \mathrm{As}_{2}$, cleaved at room temperature and measured at $T=$ 40 K, by Hsieh et al. [48] (see Fig. 3d). Their surface shows some areas of clear atomically resolved unit-cell-doubling stripes, sepa- 
rated by some indistinct areas. In addition, part of the stripe region is covered with maze-like networks. This maze-like network dominates the surface when the measurement temperature is increased to $200 \mathrm{~K}$. This indicates that surfaces of the investigated compounds are not as stable as, for example, the surface of Bi2212, where atomic resolution has been reported even at room temperature $[49,50]$.

Another configuration which may be achieved by the removal of half the $\mathrm{Ba}$ atoms is a $\sqrt{2} \times \sqrt{2}$ surface structure, if every second $\mathrm{Ba}$ row diagonal to the $a b$ lattice vectors (see Fig. 2) is removed. There exists preliminary evidence supporting this scenario [51].

\section{Tunneling Spectroscopy}

The identification of the superconducting order parameter, including the gap size and symmetry, is a necessary step to determining the microscopic superconducting mechanism. The possibility of multiple gaps, potentially with different symmetries arising from the multi-band electronic structure, has motivated intense efforts in studying the electronic structure of the pnictides with several complementary spectroscopic techniques, among them STS.

The power of STM/STS is to measure the electronic structure with sub-atomic spatial resolution. The reliability of this measurement depends on the condition of the tunnel junction. Tip preparation methods have been honed by years of STM studies, so the condition of the tunnel junction depends mainly on the sample quality and material-specific surface stability. Differential tunneling conductance spectra, $d I / d V$, measured on different pnictides will be reviewed in this section. We present some STS measurements from polycrystalline oxypnictides, then focus on the tunneling spectra of 122 single crystals.
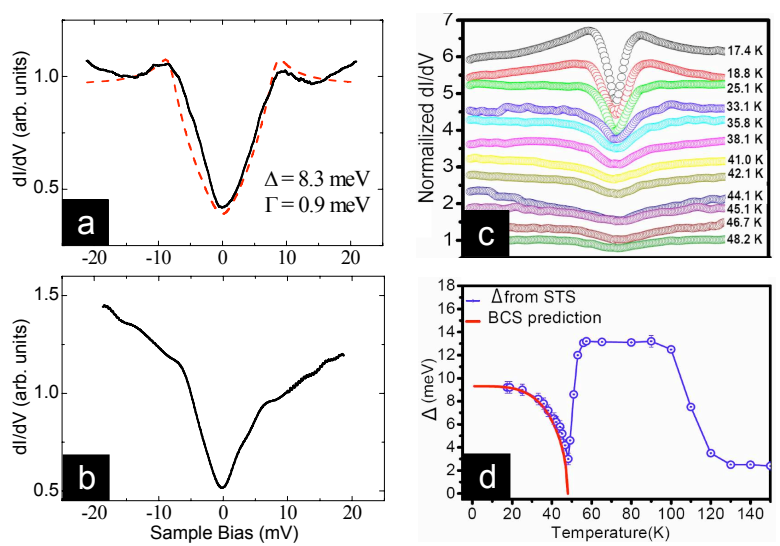

Figure 4: Single point spectra recorded on polycrystalline samples. (a-b) Spectra recorded at $4.2 \mathrm{~K}$ on $\mathrm{SmFeAsO}_{0.85}$ by Millo et al. [52]. (a) Spectrum in one of the regions where coherence peaks were observed; the red dashed line denotes a fit to a $d$ wave superconductor. (b) Spectrum recorded on the $\sim 70 \%$ of the sample surface which did not show coherence peaks. (c-d) Normalized tunneling spectra recorded by Pan et al. on $\mathrm{NdFeAsO}_{0.86} \mathrm{~F}_{0.14}$ [53]. (c) Evolution of the gap with temperature. (d) Temperature dependence of the gap magnitude compared to a thermally broadened $s$-wave BCS model.

\subsection{Point Spectra}

Two papers present tunneling spectroscopy on polycrystalline 1111 samples. Millo et al. have detected an $\sim 8 \mathrm{meV} d$ wave-like gap with clear coherence peaks in the $\mathrm{SmFeAsO}_{0.85}$ compound, with $T_{\mathrm{c}}=$ $52 \mathrm{~K}$ (Fig. 4a-b) [52]. However, $\sim 70 \%$ of the sample surface is dominated by $\bigvee$-shaped spectra with only gap-edge kinks at $7 \mathrm{meV}$, attributed to suppressed superconductivity on the sample surface or effects from a non-superconducting FeAs phase of the material. It should be noted that, even 
though different in shape, both gaps disappear above $T_{\mathrm{c}}$. The authors calculate the reduced gap $2 \Delta / k_{\mathrm{B}} T_{\mathrm{c}}=3.55-3.8$.

Pan et al. [53] present $d I / d V$ spectra measured on a $\mathrm{NdFeAsO}_{0.86} \mathrm{~F}_{0.14}$ sample with $T_{\mathrm{c}}=48 \mathrm{~K}$. A $\sim 9 \mathrm{meV}$ gap with corresponding $2 \Delta / k_{\mathrm{B}} T_{\mathrm{c}}=4.5$ is found at $17 \mathrm{~K}$. However, most of the spectra do not show sharp coherence peaks, leading to a very short lifetime in both $s$-wave and $d$-wave fitting. At low temperatures, the temperature dependence of the spectra is consistent with BCS predictions (see Fig. 4d). However, for sample temperatures above $T_{\mathrm{c}}$, a new gaplike feature of unclear origin appears with a gap width larger than the superconducting gap.

We turn now to STS data taken on cleaved single crystal surfaces. Boyer et al. have observed spectra with a $10 \mathrm{meV}$ gap on cold-cleaved $\mathrm{Sr}_{1-x} \mathrm{~K}_{x} \mathrm{Fe}_{2} \mathrm{As}_{2}$ samples with $T_{\mathrm{c}}=32 \mathrm{~K}$ [45]. The spectral shape is strongly reminiscent of the $d$-wave gap in the cuprates. The observed gap value is comparable to the $12 \mathrm{meV}$ gap in the hole-like, $\Gamma$-centered, inner $(\alpha)$ band observed in ARPES measurements on a $\mathrm{Ba}_{0.6} \mathrm{~K}_{0.4} \mathrm{Fe}_{2} \mathrm{As}_{2}$ sample with similar $T_{\mathrm{c}}$ [54]. However, the $12 \mathrm{meV}$ gap measured by ARPES is isotropic and nodeless in momentum space. ARPES observed an additional gap of $6 \mathrm{meV}$ in the hole-like, $\Gamma$-centered, outer $(\beta)$ band in this material [54].

Yin et al. [46] performed spectroscopy on an electron-doped $\mathrm{BaFe}_{1.8} \mathrm{Co}_{0.2} \mathrm{As}_{2}$ compound with $T_{\mathrm{c}}=25.3 \mathrm{~K}$, resolving only a single energy gap $\bar{\Delta}=6.25 \mathrm{meV}$. This result is compatible with measurements taken on a similar compound by Massee et al., who observe a single $\sim 7 \mathrm{meV}$ gap [47] (see Fig. 5). The spectra recorded by both groups show a clear superconducting gap structure with pronounced coherence peaks at all locations in the measurement region of the sample surface, ruling out nanoscale phase separation between superconducting and non-superconducting (magnetic) regions. Furthermore, Yin et al. prove the superconducting nature of the gap by vortex imaging, discussed in Section 5 .

The gap size of $6-7 \mathrm{meV}$ measured by Yin et al. and Massee et al. is in reasonable agreement with ARPES measurements in electron doped $\mathrm{BaFe}_{1.85} \mathrm{Co}_{0.15} \mathrm{As}_{2}$ [55]. In contrast to hole doped $\mathrm{Ba}_{0.6} \mathrm{~K}_{0.4} \mathrm{Fe}_{2} \mathrm{As}_{2}$ [54], the $\alpha$ band is located entirely below the Fermi surface, leaving behind only the $\sim 6 \mathrm{meV}$ gaps in the hole-like $\beta$ band and two other electron-like bands.
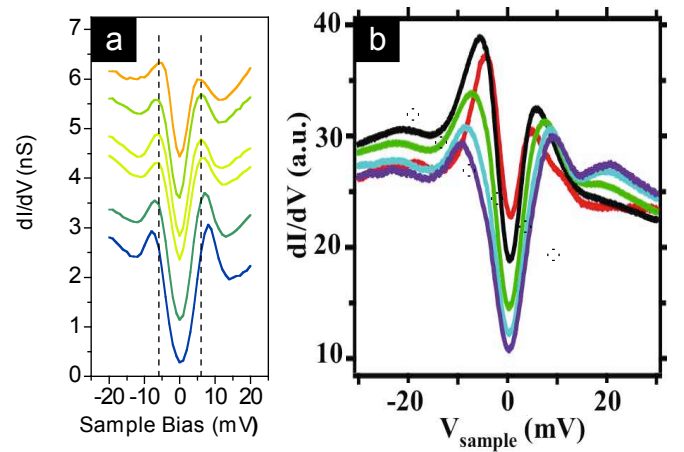

Figure 5: Spectra recorded on single crystalline $\mathrm{BaFe}_{2-x} \mathrm{Co}_{x} \mathrm{As}_{2}$. (a) Representative point spectra recorded on $\mathrm{BaFe}_{1.8} \mathrm{Co}_{0.2} \mathrm{As}_{2}$ by $\mathrm{Yin}$ et al. [46]. (b) Spectra, binned by gap size and then averaged, recorded by Massee et al. on $\mathrm{BaFe}_{1.86} \mathrm{Co}_{0.14} \mathrm{As}_{2}$ [47]. Massee et al. point out that larger gapped spectra appear to be deeper compared to smaller gapped spectra [47]; this can also be observed in spectra from Yin et al.

Although spectra vary between different STM experiments, some common features can be extracted.

(1) Most spectra have a $\bigvee$-shaped background, which is likely related to the normal state of the pnictides. This assumption is 
spectra in parent compounds [47] and in vortex cores where superconductivity is suppressed (Fig. 9b). In Bi2212, by contrast, the background is roughly linear [22].

(2) To date, no clear information about the gap symmetry can be extracted from tunneling spectra. In the polycrystalline samples, fitting to the observed weak coherence peaks requires short quasiparticle lifetimes which make $s$ and $d$-wave fits indistinguishable [53]. Furthermore, since all spectra have been recorded at or above $4.2 \mathrm{~K}$, thermal broadening makes it difficult to extract the gap symmetry [56]; nodeless $s$ wave spectra may be smeared out and appear to fit a $d$-wave model. Ultra low temperature STS measurements are therefore expected to shed light on the gap symmetry mystery. In addition, the $\bigvee$-shaped background appearing in almost all of the spectra will have to be subtracted or normalized out in some fashion before any reasonable fit can be obtained.

(3) STM spectra have until now not shown any evidence of multiple gaps. Because STM measures spectra averaged in momentum-space, the relative contribution of different bands needs to be considered. At present, it is unclear if the matrix element that describes the coupling between states in tip and sample might be different for electron or hole like pockets and whether it varies between different bands. In addition, other gaps with weak coherence peaks may be hidden within momentum-space averaged spectra.

(4) Calculated reduced gaps vary among the different experiments. For single crystal samples, values range from 5.7 to 7.4 (see Table 1) falling in the strong coupling regime. All single-crystal results exceed the values for weak coupling $s$-wave or $d$-wave BCS superconductors, which are 3.5 and
Table 1: Reduced gap in pnictide superconductors

\begin{tabular}{|c|c|c|c|c|}
\hline \hline Compound & Ref. & $T_{\mathrm{c}}$ & $\Delta$ & $\frac{2 \Delta}{k_{\mathrm{B}} T_{\mathrm{c}}}$ \\
\hline $\mathrm{SmFeAsO}_{0.85}$ & {$[52]$} & $52 \mathrm{~K}$ & $8.3 \mathrm{meV}$ & 3.7 \\
\hline $\mathrm{NdFeAsO}_{0.86} \mathrm{~F}_{0.14}$ & {$[53]$} & $48 \mathrm{~K}$ & $9.2 \mathrm{meV}$ & 4.5 \\
\hline $\mathrm{Sr}_{1-x} \mathrm{~K}_{x} \mathrm{Fe}_{2} \mathrm{As}_{2}$ & {$[45]$} & $32 \mathrm{~K}$ & $10.0 \mathrm{meV}$ & 7.3 \\
\hline $\mathrm{BaFe}_{1.8} \mathrm{Co}_{0.2} \mathrm{As}_{2}$ & {$[46]$} & $25 \mathrm{~K}$ & $6.3 \mathrm{meV}$ & 5.7 \\
\hline $\mathrm{BaFe}_{1.86} \mathrm{Co}_{0.14} \mathrm{As}_{2}$ & {$[47]$} & $22 \mathrm{~K}$ & $7.0 \mathrm{meV}$ & 7.4 \\
\hline \hline
\end{tabular}

\section{3 , respectively [57].}

\subsection{Gap maps}

With the same STM/STS techniques used in Bi2212, a map of the magnitude of the superconducting gap $\Delta$ can be obtained for the pnictides. Fig. 6a shows such a gap map taken at zero magnetic field and $6.25 \mathrm{~K}$ for $\mathrm{BaFe}_{1.8} \mathrm{Co}_{0.2} \mathrm{As}_{2}$ as recorded by Yin et al. [46].
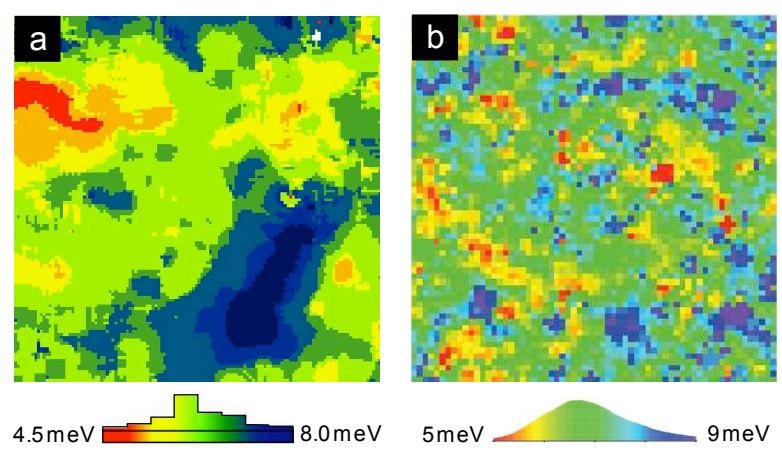

Figure 6: Gap maps recorded by Yin et al. over a $20 \times 20 \mathrm{~nm}^{2}$ area of on $\mathrm{BaFe}_{1.8} \mathrm{Co}_{0.2} \mathrm{As}_{2}$ at $6.25 \mathrm{~K}$ (a) and Massee et al. over a $18.9 \times 18.9 \mathrm{~nm}^{2}$ area of $\mathrm{BaFe}_{1.86} \mathrm{Co}_{0.14} \mathrm{As}_{2}$ at $4.2 \mathrm{~K}(\mathrm{~b})$.

The gap magnitude ranges from 4.5 to $8.0 \mathrm{meV}$ with an average of $\bar{\Delta}=6.25 \mathrm{meV} \pm$ $0.73 \mathrm{meV}$. The fractional variation in gap size is calculated to be $\sigma / \bar{\Delta}=12 \%$. Massee et al. [47] display a similar average gap of $7 \mathrm{meV}$, gap range of $5.5 \mathrm{meV}$, and fractional variation of $13 \%$ [58] in the same material, see Fig. 6b.

The gap maps of both groups reveal nm8 sized patches of different gap magnitude, 
but in the data by Massee et al., these patches are smaller in size compared to Yin et al.'s data. Massee et al. relate the nmsized length scale of the gap variations to the Co-Co separation and postulate Co doping is the source of the small gap inhomogeneity in the pnictides [47].

The zero bias conductance (ZBC) decreases with increasing gap size in the pnictides (see Fig. 5a and b). As first calculated by Massee et al., there is an anticorrelation between the magnitude of the superconducting gap and the ZBC. This behavior in pnictides may be explained by impurity scattering [59].

Both electronic homogeneity and dependance of ZBC on the gap size in the pnictides differ markedly from observations in the cuprates. While both Yin et al. and Massee et al. measure a $12-13 \%$ fractional variation in gap magnitude on their samples, the typical fractional variation reported in optimally doped $\mathrm{Bi} 2212, \sigma / \bar{\Delta}=$ $7 \mathrm{meV} / 33 \mathrm{meV} \approx 21 \%$, is almost twice this value [25]. In addition, spectra in the cuprates show larger ZBC values with increasing gap magnitude, which is the opposite behaviour to that of the pnictides [25]. In Yin et al., spectra with shallower superconducting gaps (larger ZBC) have weaker coherence peaks, which is consistent with a shorter quasiparticle lifetime, but Massee et al. show the opposite trend, which needs further investigation.

A possible resolution to the contrast between pnictides and cuprates may arise from several recent studies of the pseudogap phase in the cuprates. Using a normalization technique, Boyer et al. reported the removal of the effects of the pseudogap, obtaining a relatively homogeneous low temperature gap distribution in $\mathrm{Bi}_{2} \mathrm{Sr}_{2} \mathrm{CuO}_{6+x}$ [60]. Ma et al. studied
$\mathrm{Bi}_{2} \mathrm{Sr}_{2-x} \mathrm{La}_{x} \mathrm{CuO}_{6+\delta}$ and, due to the chosen doping, were able to measure spectra in which the energy scales of superconducting and pseudogap were distinct [61]. In these studies, the separated superconducting gap seems to show a different correlation between ZBC and gap magnitude compared to what is usually observed in the cuprates: the larger the gaps, the smaller the ZBC. Therefore, the apparent opposite relation between coherence peak height and gap width in the cuprates may be due not to the superconducting gap itself but to a competing pseudogap, which depletes the spectral weight for pairing [62].

\section{Imaging of Vortex Lattice by STS}

Vortex lattice geometry in BCS-type superconductors was first observed by Bitter decoration [63]. Subsequent application of high resolution STM/STS techniques [64, $65,66]$ revealed detailed information about the vortex internal structure [64, 34]. This information about vortex core states, as well as vortex shape and interactions between vortices, is fundamentally linked to the properties, including pairing symmetry, of the superconducting charge carriers.

\subsection{Vortex Lattice Imaging}

Vortex imaging via STS relies on the fact that within vortex cores, the quasiparticle DOS differs dramatically from the superconducting DOS outside the vortex. Since coherence peaks are significantly depressed inside the vortex core, vortices can be imaged by mapping the differential conductance at the coherence peak energy [64]. Other approaches include mapping at an energy corresponding to a vortex core state [32]. STS mapping is also sensitive to non-vortex variations in the quasiparticle DOS, caused by surface impurities, 
adsorbates, defects, or any other inhomogeneity.

Yin et al. have imaged vortices by mapping the conductance at an energy close to the superconducting coherence peaks. Fig. 7 shows the conductance maps, recorded at both $9 \mathrm{~T}$ (a) and $6 \mathrm{~T}(\mathrm{~b})$. Vortices appear as broad depressions caused by the decreased DOS at the coherence peak energy inside the vortex. The per-vortex flux is in good agreement with the expected superconducting magnetic flux quantum, $\Phi_{0}=h / 2 e$.

Much can be learned about vortex interactions and pinning by studying the locations of vortices with respect to each other and to other structures within the superconductor. Vortex interactions can be roughly categorized into three scenarios. First, in the absence of strong pinning sites within the material, the vortex-vortex interaction force dominates, and the vortices crystalize into a hexagonal lattice, shown schematically in Fig. 8a. For example, Fig. 8d shows the first STS-imaged vortex lattice in $\mathrm{NbSe}_{2}$, recorded by Hess et al. [64], revealing an almost perfect hexagonal symmetry.

In the presence of strong pinning sites, the vortex arrangement with respect to those sites depends on the anisotropy of the material. In highly anisotropic superconductors, a one-dimensional vortex line may split like a stack of pancakes into point-like objects with the freedom to move independently in each superconducting layer [67]. In this scenario, pancakes may find pinning sites independently in each layer, resulting in a high correlation between observed vortex and pinning locations in any given layer. This second scenario is shown schematically in Fig. 8b, and is realized in Bi2212 (Fig. 8e, Pan et al. [32]), where the vortices (depicted as circles of radius $30 \AA$ ) are clearly corre-

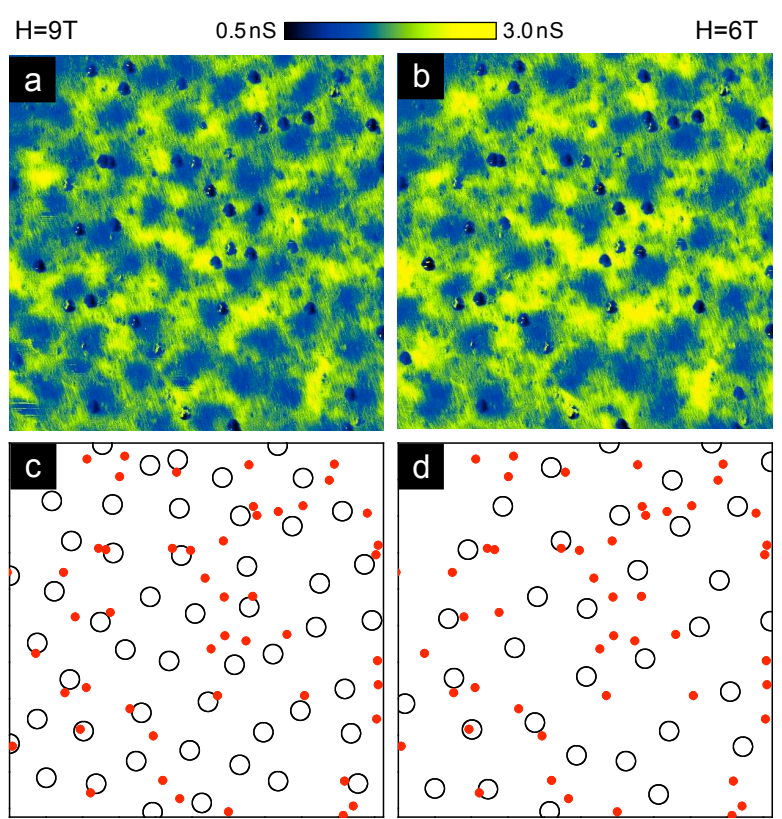

Figure 7: $100 \times 100 \mathrm{~nm}^{2}$ differential conductance maps recorded in $9 \mathrm{~T}(\mathrm{a})$ and $6 \mathrm{~T}(\mathrm{~b})$ magnetic field by Yin et al., revealing the sample DOS at $5 \mathrm{meV}$. Vortices appear as dark features due to the suppression of the coherence peaks inside the vortex core. Vortices form a disordered lattice. The average pervortex flux values of $\bar{\phi}(9 \mathrm{~T})=2.05 \times 10^{-15} \mathrm{Tm}^{2}$ and $\bar{\phi}(6 \mathrm{~T})=2.17 \times 10^{-15} \mathrm{Tm}^{2}$ are in good agreement with the expected superconducting magnetic flux quantum $\Phi_{0}=2.07 \times 10^{-15} \mathrm{Tm}^{2}$. In addition to the vortices, impurities are visible in (a) and (b), appearing as smaller, deeper coherence peak depressions. There is no statistically significant correlation between vortex and impurity locations. (c) and (d): idealized data showing both vortices (open dark circles) and impurities (red dots).

lated with the impurities (visible as small, irregular black spots).

Finally, in a more isotropic superconductor with strong pinning sites, the vortices must remain as line objects, and can bend only slightly between layers to maximize their overlap with impurities throughout the bulk. In this scenario, there may be very little observable correlation between vortex locations and impurities in any one layer. 
This third scenario is depicted schematically in Fig. 8c.

As observed by low temperature STS imaging in the cuprates [30, 33, 32], the vortices imaged by Yin et al. in $\mathrm{BaFe}_{1.8} \mathrm{Co}_{0.2} \mathrm{As}_{2}$ form a disordered lattice, indicating the presence of strong pinning sites. This observation is consistent with recent small-angle neutron scattering experiments on a similar compound, $\mathrm{BaFe}_{1.86} \mathrm{Co}_{0.14} \mathrm{As}_{2}$ [68]. In addition to the vortices, there are also sharp minima in conductance, independent of magnetic field, which may be attributed to strong scatterers near the surface. There is no correlation between the locations of the vortices and these near-surface impurities. Fig. $8 \mathrm{f}$ shows the same data as Fig. 7 but with vortex cores emphasized as circles of radius the coherence length. At $9 \mathrm{~T}$, roughly $10.6 \%$ of the field of view is covered by vortex cores and 4 out of 49 impurities lie within a vortex core. This number corresponds to $8.2 \%$, which is not a statistically significant deviation from $10.6 \%$. This analysis proves that bulk pinning, and not surface pinning, must play a significant role in this superconductor.

\subsection{Vortex Spectroscopy}

Microscopic calculations of vortex core states predict different spectral features depending on the pairing symmetry of the superconductor. Vortex core states in conventional $s$-wave superconductors are expected to show a ZBC peak, caused by tunneling of electrons occupying states bound to the vortex core $[69,70,71,72]$. This effect is demonstrated in Fig. 9a by DOS spectra measured in the vortex core of $\mathrm{NbSe}_{2}$, which show a pronounced ZBC peak. It is important to note that the ZBC peak is observed only in clean superconductors, where the
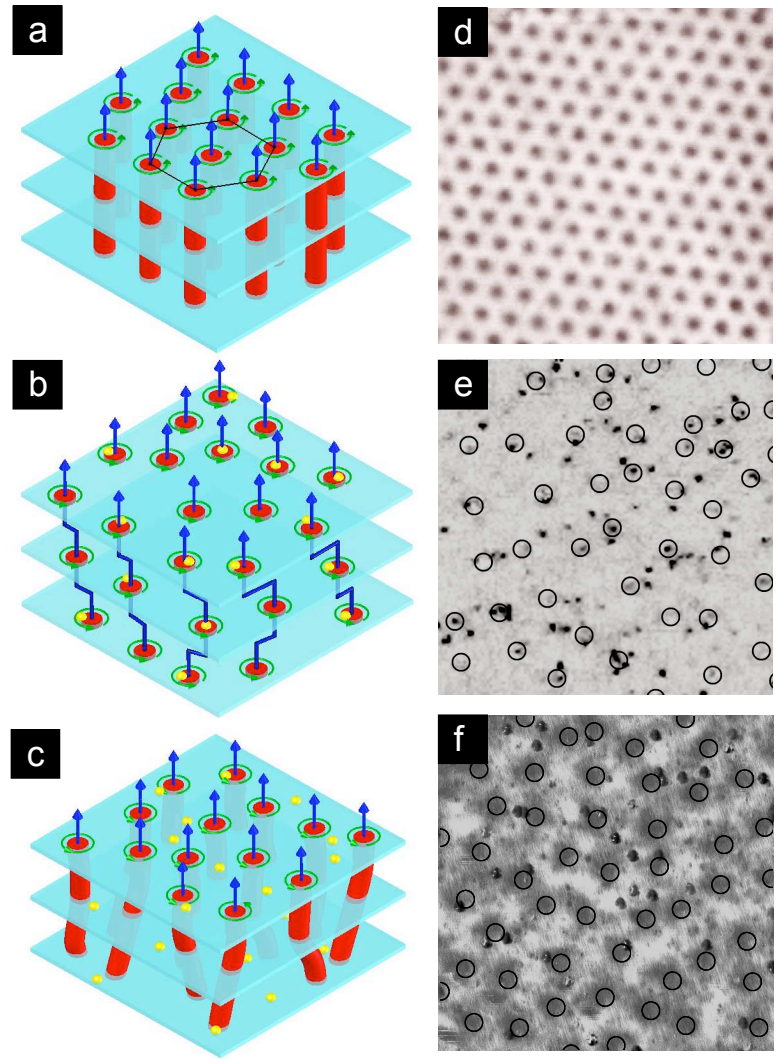

Figure 8: (a-c) Three different vortex interaction regimes. (a) Vortices in a clean superconductor. Both surface and bulk pinning are absent and the vortices arrange in a perfect hexagonal Abrikosov lattice due to the repulsion between vortices. (b) Pancake vortices in a superconductor with weak interlayer coupling. Some of the pancake vortices are pinned to impurities (yellow dots) within the layers and marked correlation between vortex and impurity locations is observed. Between the layers, pancake vortices are connected by Josephson vortices (blue lines). (c) Vortices in a superconductor where bulk pinning dominates. The flux penetrates the sample in a tubular fashion, but no hexagonal lattice can be formed as vortices are pinned by bulk defects. There is no correlation between surface defects and vortex locations. (d-f) Examples of these three scenarios, imaged by STS (d) in $\mathrm{NbSe}_{2}$ by Hess et al. [64] in $1 \mathrm{~T}$ magnetic field at $1.8 \mathrm{~K}$, (e) in $\mathrm{Bi}_{2} \mathrm{Sr}_{2} \mathrm{CaCu}_{2} \mathrm{O}_{8+\delta}$ by Pan et al. [32] in $7 \mathrm{~T}$ magnetic field at $4.2 \mathrm{~K}$, and (f) in $\mathrm{BaFe}_{1.8} \mathrm{Co}_{0.2} \mathrm{As}_{2}$ by Yin et al. [46] in $9 \mathrm{~T}$ magnetic field at $6.25 \mathrm{~K}$.

electronic mean free path $\ell$ is much larger than the coherence length $\xi$. In this partic- 
ular sample of $\mathrm{NbSe}_{2}$ [73], $\ell=690 \AA$ and $\xi=77 \AA$, putting it clearly in the regime of clean superconductors. For dirty superconductors, however, there is no ZBC peak and one observes instead an almost flat DOS, due to quasiparticle scattering [65].
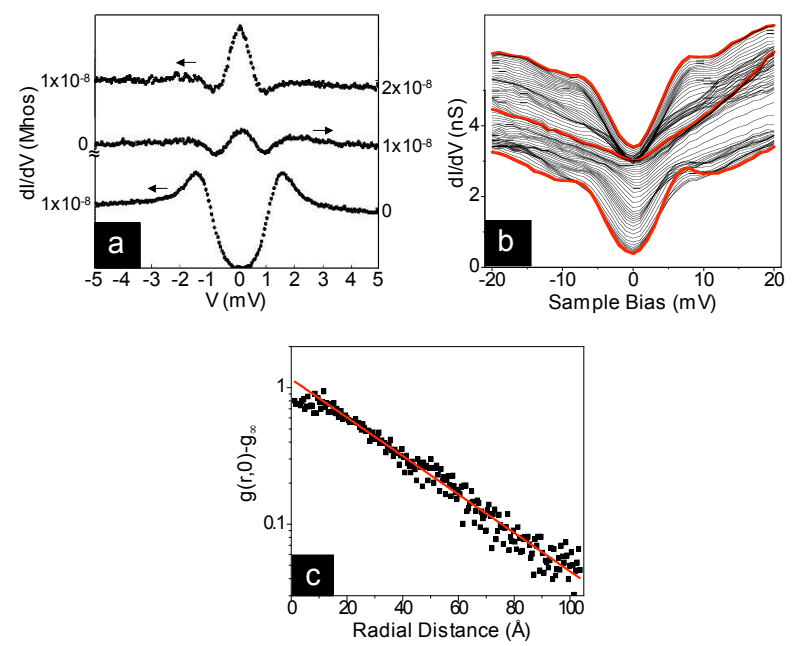

Figure 9: Comparison between vortex core spectra taken on a conventional s-wave superconductor and a pnictide superconductor. (a) $d I / d V$ spectra in $0.02 \mathrm{~T}$ magnetic field at $1.85 \mathrm{~K}$ in $\mathrm{NbSe}_{2}$ $\left(T_{\mathrm{c}}=7.2 \mathrm{~K}\right)$, taken at a vortex center (uppermost spectrum), $75 \AA$ away from a vortex core (middle spectrum), and $2000 \AA$ away form the vortex core by Hess et al. [64]. (b) A $14.5 \mathrm{~nm}$ long linecut of $d I / d V$ spectra taken through a vortex location in $\mathrm{BaFe}_{1.8} \mathrm{Co}_{0.2} \mathrm{As}_{2}$, showing the smooth evolution from the off-vortex DOS with pronounced coherence peaks to the on-vortex DOS with suppressed coherence peaks and no ZBC peak. The data were taken at $9 \mathrm{~T}$ magnetic field and $T=6.15 \mathrm{~K}$ by Yin et al. (c) Azimuthally averaged radial dependence of the differential conductance $g(r, 0)$ around a single vortex as measured (black squares) and fit by an exponential decay (red line) by Yin et al. The constant background $g_{\infty}$ has been removed in order to emphasize the exponential decay on the logarithmic scale of the $y$-axis. The exponential fit leads to an average coherence length of $\xi=27.6 \AA$ with standard deviation $2.9 \AA$ for all vortices investigated.

In contrast to conventional $s$-wave superconductors in the clean limit, the conduc- tance measured by Yin et al. at the vortex center in $\mathrm{BaFe}_{1.8} \mathrm{Co}_{0.2} \mathrm{As}_{2}$ shows only an enhanced sub-gap density of states but no ZBC peak. This is apparent in Fig. 9b where a linecut through one of the vortices at $9 \mathrm{~T}$ magnetic field is displayed.

The spatial dependence of the vortex spectroscopy also enables the first direct measure of the superconducting coherence length in this material. By fitting an exponential decay to the decrease of ZBC from the value at the vortex center to the value far away from the vortex, a coherence length of $\xi=27.6 \AA$ is revealed (see Fig. 9c). This corresponds to an upper critical field of $H_{\mathrm{c} 2}=43 \mathrm{~T}$, calculated from the GinzburgLandau expression $H_{\mathrm{c} 2}=\Phi_{0} / 2 \pi \xi^{2}$.

Since the compound under investigation has a mean free path that is three times larger than the coherence length [46], it is considered to be a clean superconductor and therefore the lack of sub-gap peaks cannot be explained by scattering effects and remains an open question.

\section{Conclusion}

Although research in the pnictides is still in its early stages, there exist some significant achievements by STM/STS on these compounds. To date STM/STS studies have contributed information about the cleaved surface structure, nature of the superconducting gap, and vortex core states. Investigations of numerous surfaces have revealed a stripe feature in almost all 122 compounds, both doped and undoped. $d I / d V$ spectra of doped single crystals show a clear superconducting gap with pronounced coherence peaks. In addition to single point spectra, maps of the gap distribution have been displayed by two different groups on comparable single crystalline 122 
samples, providing information about the spatial inhomogeneity of the gap. From vortex imaging, a short coherence length has been directly measured for the first time by any technique.

Looking forward, there are several problems that remain to be addressed by STM/STS. The biggest roadblock to further STM/STS studies is surface characterization. Surface morphologies have been observed to depend sensitively on cleaving conditions. In addition, surface instability and polar cleavage planes are complex and unsolved problems which have made it very difficult to achieve repeatable atomic resolution topographies on these compounds.

Another key issue is the gap symmetry. Single point spectra at ultra-low temperatures would allow better fitting without the complications of thermal broadening. A different approach to extract the gap symmetry is to probe the local DOS around both magnetic and non-magnetic impurities, which has been successfully applied to the cuprates [27, 28]. Similar experiments in the pnictides, and comparison to theory [74], could address the gap symmetry question.

In addition, momentum space information might be accessed by Fourier transforming DOS maps and extracting quasiparticle interference (QPI) patterns [34]. The QPI patterns should be used to explore the pairing symmetry as proposed in [75]: in the case of dominant non-magnetic scatterers, a detected absence of interference peaks surrounding the reciprocal lattice vector would be evidence of the out-of-phase extended $s$-wave pairing.

In conclusion, there is much potential for significant contributions by STM/STS to resolve the most important open questions in pnictide superconductivity, but ad- ditional work is needed to overcome surfacerelated challenges.

\section{Acknowledgments}

We thank Mark Golden, David Hsieh, Eric Hudson, Donghui Lu, Freek Massee, Kinsei Terashima, Ali Yazdani, and Ming Yi for stimulating discussions and Barbara Drauschke for administrative assistance. The authors acknowledge support by the NSF (DMR-0508812) and the AFOSR (FA9550-05-1-0371). T.L.W. acknowledges support from an NDSEG fellowship.

\section{References}

[1] Y. Kamihara, T. Watanabe, M. Hirano, H. Hosono, J. Am. Chem. Soc. 130 (2008) 3296.

[2] H. Takahashi, K. Igawa, K. Arii, Y. Kamihara, M. Hirano, H. Hosono, Nature 453 (2008) 376.

[3] X. H. Chen, T. Wu, G. Wu, R. H. Liu, H. Chen, D. F. Fang, Nature 453 (2008) 761.

[4] G. F. Chen, Z. Li, D. Wu, G. Li, W. Z. Hu, J. Dong, P. Zheng, J. L. Luo, N. L. Wang, Phys. Rev. Lett. 100 (2008) 247002.

[5] Z.-A. Ren, G.-C. Che, X.-L. Dong, J. Yang, W. Lu, W. Yi, X.-L. Shen, Z.-C. Li, L.-L. Sun, F. Zhou, Z.-X. Zhao, Europhys. Lett. 83 (2008) 17002.

[6] H. Wen, G. Mu, L. Fang, H. Yang, X. Zhu, Europhys. Lett. 82 (2008) 17009.

[7] C. Wang, L. Li, S. Chi, Z. Zhu, Z. Ren, Y. Li, Y. Wang, X. Lin, Y. Luo, S. Jiang, X. Xu, G. Cao, Z. Xu, Europhys. Lett. 83 (2008) 6700.

[8] M. Rotter, M. Tegel, D. Johrendt, Phys. Rev. Lett. 101 (2008) 107006.

[9] N. Ni, S. L. Bud'ko, A. Kreyssig, S. Nandi, G. E. Rustan, A. I. Goldman, S. Gupta, J. D. Corbett, A. Kracher, P. C. Canfield, Phys. Rev. B 78 (2008) 014507.

[10] K. Sasmal, B. Lv, B. Lorenz, A. M. Guloy, F. Chen, Y. Y. Xue, C. Chu, Phys. Rev. Lett. 101 (2008) 107007.

[11] G. F. Chen, Z. Li, G. Li, W. Z. Hu, J. Dong, J. Zhou, X. Zhang, P. Zheng, N. Wang, J. Luo, Chin. Phys. Lett. 25 (2008) 3403. 
[12] T. Park, E. Park, H. Lee, T. Klimczuk, E. D. Bauer, F. Ronning, J. D. Thompson, J. Phys.: Condens. Matter 20 (2008) 322204.

[13] G. Wu, H. Chen, T. Wu, Y. L. Xie, Y. J. Yan, R. H. Liu, X. F. Wang, J. J. Ying, X. H. Chen, J. Phys.: Condens. Matter 20 (2008) 422201.

[14] M. Torikachvili, S. Bud'ko, N. Ni, P. Canfield, Phys. Rev. Lett. 101 (2008) 057006.

[15] H. S. Jeevan, Z. Hossain, D. Kasinathan, H. Rosner, C. Geibel, P. Gegenwart, Phys. Rev. B 78 (2008) 092406.

[16] X. F. Wang, T. Wu, G. Wu, H. Chen, Y. L. Xie, J. J. Ying, Y. J. Yan, R. H. Liu, X. H. Chen, Phys. Rev. Lett. 102 (2009) 117005.

[17] N. D. Zhigadlo, S. Katrych, Z. Bukowski, S. Weyeneth, R. Puzniak, J. Karpinski, J. Phys.: Condens. Matter 20 (2008) 342202.

[18] R. Prozorov, M. E. Tillman, E. D. Mun, P. C. Canfield, New J. Phys. 11 (2009) 035004.

[19] A. S. Sefat, R. Jin, M. A. McGuire, B. C. Sales, D. J. Singh, D. Mandrus, Phys. Rev. Lett. 101 (2008) 117004.

[20] L. J. Li, Q. B. Wang, Y. K. Luo, H. Chen, Q. Tao, Y. K. Li, X. Lin, M. He, Z. W. Zhu, G. H. Cao, Z. A. Xu, New J. Phys. 11 (2009) 025008.

[21] J. M. Tarascon, L. H. Greene, P. Barboux, W. R. McKinnon, G. W. Hull, T. P. Orlando, K. A. Delin, S. Foner, E. J. McNiff, Phys. Rev. B 36 (1987) 8393.

[22] O. Fischer, M. Kugler, I. Maggio-Aprile, C. Berthod, C. Renner, Rev. Mod. Phys. 79 (2007) 353.

[23] S. H. Pan, J. P. O’Neal, R. L. Badzey, C. Chamon, H. Ding, J. R. Engelbrecht, Z. Wang, H. Eisaki, S. Uchida, A. K. Gupta, K. Ng, E. W. Hudson, K. M. Lang, J. C. Davis, Nature 413 (2001) 282.

[24] K. M. Lang, V. Madhavan, J. E. Hoffman, E. W. Hudson, H. Eisaki, S. Uchida, J. C. Davis, Nature 415 (2002) 412.

[25] K. McElroy, D.-H. Lee, J. E. Hoffman, K. M. Lang, J. Lee, E. W. Hudson, H. Eisaki, S. Uchida, J. C. Davis, Phys. Rev. Lett. 94 (2005) 197005.

[26] K. K. Gomes, A. N. Pasupathy, A. Pushp, S. Ono, Y. Ando, A. Yazdani, Nature 447 (2007) 569.

[27] S. H. Pan, E. W. Hudson, K. M. Lang, H. Eisaki, S. Uchida, J. C. Davis, Nature 403 (2000) 746.
[28] E. W. Hudson, K. M. Lang, V. Madhavan, S. H. Pan, H. Eisaki, S. Uchida, J. C. Davis, Nature 411 (2001) 920.

[29] K. Chatterjee, M. C. Boyer, W. D. Wise, T. Kondo, T. Takeuchi, H. Ikuta, E. W. Hudson, Nat. Phys. 4 (2008) 108.

[30] I. Maggio-Aprile, C. Renner, A. Erb, E. Walker, O. Fischer, Phys. Rev. Lett. 75 (1995) 2754.

[31] C. Renner, B. Revaz, K. Kadowaki, I. MaggioAprile, O. Fischer, Phys. Rev. Lett. 80 (1998) 3606.

[32] S. H. Pan, E. W. Hudson, A. K. Gupta, K. W. Ng, H. Eisaki, S. Uchida, J. C. Davis, Phys. Rev. Lett. 85 (2000) 1536.

[33] B. W. Hoogenboom, K. Kadowaki, B. Revaz, M. Li, C. Renner, O. Fischer, Phys. Rev. Lett. 87 (2001) 267001.

[34] J. E. Hoffman, E. W. Hudson, K. M. Lang, V. Madhavan, H. Eisaki, S. Uchida, J. C. Davis, Science 295 (2002) 466.

[35] J. G. Simmons, J. Appl. Phys. 34 (1963) 1793.

[36] D. J. Derro, E. W. Hudson, K. M. Lang, S. H. Pan, J. C. Davis, J. T. Markert, A. L. de Lozanne, Phys. Rev. Lett. 88 (2002) 097002.

[37] I. R. Shein, A. L. Ivanovskii, arXiv:0806.0750, 2008.

[38] J. C. Wojdet, I. de P. R. Moreira, F. Illas, J. Am. Chem. Soc. 131 (2009) 906.

[39] D. H. Lu, M. Yi, S.-K. Mo, A. S. Erickson, J. Analytis, J.-H. Chu, D. J. Singh, Z. Hussain, T. H. Geballe, I. R. Fisher, Z.-X. Shen, Nature 455 (2008) 81.

[40] M. Hossain, J. Mottershead, D. Fournier, A. Bostwick, J. L. McChesney, E. Rotenberg, R. Liang, W. N. Hardy, G. A. Sawatzky, I. Elfimov, D. A. Bonn, A. Damascelli, Nat. Phys. 4 (2008) 527.

[41] R. Hesper, L. H. Tjeng, A. Heeres, G. A. Sawatzky, Phys. Rev. B 62 (2000) 16046.

[42] Q. Huang, Y. Qiu, W. Bao, M. A. Green, J. W. Lynn, Y. C. Gasparovic, T. Wu, G. Wu, X. H. Chen, Phys. Rev. Lett. 101 (2008) 257003.

[43] S. de Jong, Y. Huang, R. Huisman, F. Massee, S. Thirupathaiah, M. Gorgoi, R. Follath, J. B. Goedkoop, M. S. Golden, arXiv:0901.2691, 2009.

[44] M. Yi, L.H. Lu, private communication, 2009.

[45] M. C. Boyer, K. Chatterjee, W. D. Wise, G. F. Chen, J. L. Luo, N. L. Wang, E. W. Hudson, arXiv:0806.4400, 2008. 
[46] Y. Yin, M. Zech, T. L. Williams, X. F. Wang, G. Wu, X. H. Chen, J. E. Hoffman, Phys. Rev. Lett. 102 (2009) 097002.

[47] F. Massee, Y. Huang, R. Huisman, S. de Jong, J. B. Goedkoop, M. S. Golden, arXiv:0812.4539, 2008.

[48] D. Hsieh, Y. Xia, L. Wray, D. Qian, K. Gomes, A. Yazdani, G. F. Chen, J. L. Luo, N. L. Wang, M. Z. Hasan, arXiv:0812.2289, 2008.

[49] M. D. Kirk, J. Nogami, A. A. Baski, D. B. Mitzi, A. Kapitulnik, T. H. Geballe, C. F. Quate, Science 242 (1988) 1673.

[50] X. L. Wu, Z. Zhang, Y. L. Wang, C. M. Lieber, Science 248 (1990) 1211.

[51] S. H. Pan, A. Li, D. R. Jayasundara, Y. Xuan, J. P. O'Neal, R. Jin, E. W. Plummer, A. S. Sefat, M. A. McGuire, B. C. Sales, D. Mandrus, http://meetings.aps.org/Meeting/MAR09/ Event/93579, 2009.

[52] O. Millo, I. Asulin, O. Yuli, I. Felner, Z. Ren, X. Shen, G. Che, Z. Zhao, Phys. Rev. B 78 (2008) 092505.

[53] M. H. Pan, X. B. He, G. Li, J. F. Wendelken, R. Jin, A. S. Sefat, M. A. McGuire, B. C. Sales, D. Mandrus, E. W. Plummer, arXiv:0808.0895, 2008.

[54] H. Ding, P. Richard, K. Nakayama, K. Sugawara, T. Arakane, Y. Sekiba, A. Takayama, S. Souma, T. Sato, T. Takahashi, Z. Wang, X. Dai, Z. Fang, G. F. Chen, J. L. Luo, N. L. Wang, Europhys. Lett. 83 (2008) 4700.

[55] K. Terashima, Y. Sekiba, J. H. Bowen, K. Nakayama, T. Kawahara, T. Sato, P. Richard, Y. M. Xu, L. J. Li, G. H. Cao, Z. A. $\mathrm{Xu}$, H. Ding, T. Takahashi, arXiv:0812.3704, 2008.

[56] The thermal broadening of recorded $d I / d V$ spectra at $4.2 \mathrm{~K}$ is $\Delta E \approx 4 k_{\mathrm{B}} T=1.4 \mathrm{meV}$.

[57] H. Won, K. Maki, Phys. Rev. B 49 (1994) 1397.

[58] F. Massee, M. Golden, private communication, 2009.

[59] G. Preosti, H. Kim, P. Muzikar, Phys. Rev. B 50 (1994) 1259.

[60] M. C. Boyer, W. D. Wise, K. Chatterjee, M. Yi, T. Kondo, T. Takeuchi, H. Ikuta, E. W. Hudson, Nat. Phys. 3 (2007) 802.

[61] J.-H. Ma, Z.-H. Pan, F. C. Niestemski, M. Neupane, Y.-M. Xu, P. Richard, K. Nakayama, T. Sato, T. Takahashi, H.-Q. Luo, L. Fang, H.-H. Wen, Z. Wang, H. Ding, V. Madhavan,
Phys. Rev. Lett. 101 (2008) 207002.

[62] T. Kondo, R. Khasanov, T. Takeuchi, J. Schmalian, A. Kaminski, Nature 457 (2009) 296.

[63] U. Essmann, H. Traeuble, Phys. Lett. A 24 (1967) 526.

[64] H. F. Hess, R. B. Robinson, R. C. Dynes, J. M. Valles, J. V. Waszczak, Phys. Rev. Lett. 62 (1989) 214.

[65] C. Renner, A. D. Kent, P. Niedermann, O. Fischer, F. Lévy, Phys. Rev. Lett. 67 (1991) 1650.

[66] S. Behler, M. Bernasconi, P. Jess, R. Hofer, H. J. Güntherodt, G. Wirth, J. Wiesner, Z. Phys. B: Condens. Matter 94 (1993) 3.

[67] J. R. Clem, Phys. Rev. B 43 (1991) 7837.

[68] M. R. Eskildsen, L. Y. Vinnikov, T. D. Blasius, I. S. Veshchunov, T. M. Artemova, J. M. Densmore, C. D. Dewhurst, N. Ni, A. Kreyssig, S. L. Bud'ko, P. C. Canfield, A. I. Goldman, Phys. Rev. B 79 (2009) 100501R.

[69] C. Caroli, P. De Gennes, J. Matricon, Phys. Lett. 9 (1964) 307.

[70] C. Caroli, J. Matricon, Z. Phys. B: Condens. Matter 3 (1965) 380.

[71] J. D. Shore, M. Huang, A. T. Dorsey, J. P. Sethna, Phys. Rev. Lett. 62 (1989) 3089.

[72] F. Gygi, M. Schluter, Phys. Rev. B 41 (1990) 822.

[73] P. de Trey, S. Gygax, J. Jan, J. Low. Temp. Phys. 11 (1973) 421.

[74] M. Lawler, E.-A. Kim, private communication, 2009.

[75] F. Wang, H. Zhai, D. Lee, Europhys. Lett. 85 (2009) 37005. 